\title{
Perioperative Microbiologic Monitoring of Sputum on Postoperative Day One as a Predictor of Pneumonia After Hepatectomy
}

\author{
Kazuhiko Sakamoto ${ }^{1}$ - Takao Tamesa ${ }^{1} \cdot$ Yoshihiro Tokuhisa $^{1} \cdot$ Satoshi Matsukuma $^{1}$. \\ Yukio Tokumitsu $^{1}$ - Yoshinari Maeda ${ }^{1}$ - Shigeru Takeda ${ }^{1} \cdot$ Tomio Ueno $^{1}$ • \\ Shigeru Yamamoto ${ }^{1} \cdot$ Shigefumi Yoshino $^{1} \cdot$ Shoichi Hazama $^{1} \cdot$ Hiroaki Nagano $^{1}$. \\ Masaaki Oka ${ }^{1}$
}

Received: 15 April 2015 / Accepted: 28 May 2015 / Published online: 24 June 2015

(C) 2015 The Author(s). This article is published with open access at Springerlink.com

\begin{abstract}
Background The purpose of this study was to retrospectively evaluate microbial examination of sputum on postoperative day one (POD1) and to determine risk factors for postoperative pneumonia (POP) after hepatectomy.

Methods Two hundred ninety-four patients who expectorated sputum on POD1 after hepatectomy between 2003 and 2014 were investigated. Sputum samples were submitted for microbial examination. Risk factors for POP were identified using multivariable analysis.

Results One hundred fifty-eight (53.7\%) of 294 patients had bacteria in their sputum on POD1. POP was observed in 24 (8.2 \%) patients, with increased mortality in the patients with POP $(0.74$ vs $12.5 \%, p<0.01)$. Multivariate analysis demonstrated that a Brinkman index of $>400$ and bacteria in sputum on POD1 were independent risk factors for POP. Bacterial homology in sputum obtained on POD1 and onset day of POP was found in 13 of the 24 (54.2\%) patients with POP. In particular, in 13 patients with POP caused by methicillin-resistant Staphylococcus aureus or Pseudomonas aeruginosa, homology was confirmed in 9 patients $(69.2 \%)$.

Conclusion A Brinkman index $\geq 400$ and bacteria in sputum on POD1 increased the risk of POP. Presence of bacteria in sputum on POD1 may be useful in determining early treatment against POP after hepatectomy.
\end{abstract}

Keywords Postoperative pneumonia $\cdot$ Hepatectomy

\section{Introduction}

Postoperative complications, such as pneumonia, aspiration, and acute respiratory distress syndrome, are usually related to the preoperative status of the patient. These postoperative pulmonary complications after surgery are associated with morbidity and have been shown to increase the hospital length-of-

Kazuhiko Sakamoto

kazusaka@yamaguchi-u.ac.jp

1 Department of Digestive Surgery and Surgical Oncology, Yamaguchi University Graduate School of Medicine, 1-1-1, Minami-Kogushi, Ube, Yamaguchi 755-8505, Japan stay. ${ }^{1,2}$ The reported incidence of postoperative pulmonary complications varies between 10 and $80 \%{ }^{3}$ With respect to postoperative pneumonia (POP) after hepatectomy, the rate has been reported as between 9.2 and $13 \% .^{4-7}$ Perioperative strategies have been introduced for the purpose of reducing postoperative pulmonary complications. ${ }^{8,9}$ Carrel et al. reported that early pneumonia after coronary artery bypass grafting (CABG) is most probably caused by microorganisms that colonize the respiratory tract before operation, and the risk of early pneumonia after $\mathrm{CABG}$ is higher in patients with positive preoperative tracheal aspirates than in those with negative preoperative tracheal aspirates. ${ }^{10}$ With reference to this report by Carrel et al., we have been performing microbial examination of sputum on postoperative day (POD) 1 .

The aims of the present study were to assess the incidence of bacteria in sputum on POD1, assess the predictive value of bacteria-positive sputum on POD1 for the occurrence of POP after hepatectomy, clarify whether bacteria-positive sputum 
on POD1 correlates with the results of bacteriology analysis performed after the onset of POP, and evaluate risk factors for the development of POP after hepatectomy.

\section{Methods}

\section{Patients}

We conducted a retrospective analysis of 363 patients undergoing hepatectomy without laparoscopy or simultaneous procedures such as biliary reconstruction, gastrointestinal resection, or splenectomy between 2003 and 2014 in the Department of Digestive Surgery and Surgical Oncology, Yamaguchi University Graduate School of Medicine. Sixty-nine patients who were not able to expectorate sputum on POD1 were excluded; therefore, 294 patients who expectorated sputum on POD1 were investigated. Written informed consent was obtained from all study patients.

\section{Preoperative Respiratory Management and Assessment}

At the first visit to our hospital, all patients were instructed to quit smoking, and respiratory rehabilitation was started at the same time. History of smoking, cigarette consumption, and Brinkman index were noted. Brinkman index was defined by the number of cigarettes smoked/day multiplied by the number of years. ${ }^{11}$ Respiratory function including vital capacity (VC) and forced expiratory volume in $1.0 \mathrm{~s}$ (FEV1.0) were measured by spirometry preoperatively.

\section{Surgical Procedures}

Intermittent pedicle clamping (Pringle maneuver) or selective clamping of the pedicles for the segment to be resected was performed only in cases of significant bleeding. Parenchymal transection was performed using cautery with irrigation forceps (CIF) between 2003 and 2006. ${ }^{12}$ We introduced an electrosurgical device (VIO 300D; ERBE Elektromedizin, Tübingen, Germany) containing the monopolar softcoagulation and bipolar clamp coagulation systems beginning in $2007 .{ }^{13,14}$

\section{Postoperative Management}

As prophylactic antibiotics, the patients received firstgeneration cephem antibiotics by intravenous infusion for $30 \mathrm{~min}$ before the operation. An additional dose was administered if the operation was prolonged beyond $3 \mathrm{~h}$. Additionally, the patients continued to receive the same antibiotics plus further treatment at 12 -h intervals for a total of $1-3$ days. After induction of anesthesia, a nasogastric tube was placed until the morning of POD1 in all patients.
Sputum samples were submitted for microbial examination (Gram stain and semiquantitative bacteriologic cultures) on POD1. Chest X-rays were performed on POD1 and POD4 routinely. For the patients suspected to have postoperative pneumonia, chest X-rays and computed tomography (CT) scans were obtained, and sputum specimens were submitted immediately for microbial examination at that time.

\section{Definition of Postoperative Pneumonia}

POP was diagnosed if purulent sputum was collected and yielded growth of relevant pathogens on culture, and if at least two of the following additional criteria were met: (1) white blood cell count $>10,000 / \mathrm{mm}^{3}$ (2) temperature $>38^{\circ} \mathrm{C}$, and (3) new or increasing lung infiltrate on conventional chest $\mathrm{X}$ ray.

\section{Statistical Analysis}

Hepatic fibrosis staging was classified by the pathologists as follows: no fibrosis (F0), portal fibrosis without septa (F1), portal fibrosis with few septa (F2), numerous septa without cirrhosis (F3), and cirrhosis (F4). ${ }^{15}$ To identify the perioperative risk factors for POP, the following variables were included: 9 patient variables (age, sex, body mass index (BMI), fibrosis staging, glucose intolerance, indocyanine green retention rate at $15 \mathrm{~min}$ (ICG-R15), VC, FEV, and sputum on POD1) and 3 surgical variables (type of hepatectomy, operation time, and blood loss). Continuous data were expressed as median and range values and were analyzed using the MannWhitney $U$ test. Categorical data were analyzed using the $\chi^{2}$ test. Variables with $p<0.05$ in the univariate analysis that were potentially predictive of POP were then entered into the multivariate logistic regression model. The cut-off value of continuous variables was determined using receiver operating characteristic (ROC) curves, and the optimal cut-off points were determined using the minimum distance from the upper-left corner to any point on the ROC curve. The odds ratio (OR) and $95 \%$ confidence interval (CI) were also calculated. A value of $p<0.05$ was considered statistically significant. All statistical calculations were performed with the IBM SPSS Statistics version 22.0 software package (IBM Japan Inc., Tokyo, Japan).

\section{Results}

Among the 294 study patients, the median age was 69 years (range, 32-85 years) and the proportion of male patients was $78.2 \%(n=230)$. The majority of patients had hepatocellular carcinoma ( $n=231: 78.6 \%$ ). Metastatic liver tumor was present in $38(12.9 \%)$ patients. A smoking history was confirmed in 198 (67.3\%) patients, and the median Brinkman index was 
Table 1 Result of microbial examination of sputum on postoperative day $1(n=158)^{\mathrm{a}}$

\begin{tabular}{lrlrlr}
\hline Gram-negative bacteria & \multicolumn{3}{l}{ Gram-positive bacteria } & \multicolumn{2}{l}{ Fungi } \\
\hline Haemophilus spp & 33 & Staphylococcus spp & 52 & Candida albicans & 36 \\
Pseudomonas aeruginosa & 19 & (MSSA) & $(38)$ & Candida glabrate & 18 \\
Enterobacter spp & 16 & (MRSA) & $(13)$ & Candida tropicalis & 6 \\
Klebsiella spp & 12 & Streptococcus constellatus & 19 & Penicillium spp & 1 \\
Acinetobacter spp & 7 & Enterococcus spp & 8 & \\
Serratia marcescens & 4 & & & \\
Branhamella catarrhalis & 2 & & & \\
Escherichia coli & 2 & & & \\
Neisseria subflava & 2 & & & \\
Stenotrophomonas maltophilia & 2 & & & \\
Bordetella bronchiseptica & 1 & & & \\
Capnocytophaga spp & 1 & & & \\
Eikenella corrodens & 1 & & & \\
Kingella denitrificans & 1 & & & \\
Kluyvera ascorbata & 1 & & & & \\
Morganella morganii & 1 & & & & \\
Proteus vulgaris & 1 & & & & \\
\hline
\end{tabular}

MSSA methicillin-sensitive Staphylococcus aureus, MRSA methicillin-resistant Staphylococcus aureus

${ }^{\text {a }}$ There are duplicated cases
405 (range, 0-2200). The median $\mathrm{VC}$ and FEV1.0 were $3.33 \mathrm{~L}$ (range, $1.28-6.14 \mathrm{~L}$ ) and $2.47 \mathrm{~L}$ (range, 0.72$4.82 \mathrm{~L})$, respectively.

One hundred fifty-eight (53.7 \%) of 294 patients had bacteria present in their sputum on POD1 (Table 1). Haemophilus species in Gram-negative bacteria, Staphylococcus species in
Gram-positive bacteria, and Candida albicans among fungi were the most commonly isolated organisms. Pseudomonas aeruginosa and methicillin-resistant Staphylococcus aureus (MRSA) were confirmed in 19 and 13 patients, respectively.

POP was observed after hepatectomy in $24(8.2 \%)$ patients (Table 2). POP was predominantly caused by P. aeruginosa (5

Table 2 Characteristics of postoperative pneumonia

Postoperative pneumonia $(n=24)$

Median duration from operation to diagnosis of POP (day)

Microbial examination of sputum on onset day of pneumonia ${ }^{a}$

Gram-negative bacteria

Gram-positive bacteria

Fungi

Bacterial homology in sputum obtained on POD1 and onset day of pneumonia

\section{$5(1-44)$}

Pseudomonas aeruginosa $\quad 5$

Enterobacter spp 3

Acinetobacter spp 2

Klebsiella spp $\quad 2$

Citrobacter freundii

Proteus mirabilis $\quad 1$

Serratia marcescens $\quad 1$

Staphylococcus spp 10

(MRSA) (8)

(MRSE)

Enterococcus spp 2

Streptococcus spp $\quad 2$

Candida albicans

unknown 2

$13(54.2 \%)$

Data are presented as absolute numbers or median (range, minimum-maximum)

POP postoperative pneumonia, MRSA methicillin-resistant Staphylococcus aureus, MRSE methicillin-resistant Staphylococcus epidermidis, POD postoperative day

${ }^{a}$ There are duplicated cases 
Table 3 Patient characteristics with and without postoperative pneumonia

\begin{tabular}{|c|c|c|c|c|c|}
\hline \multirow[b]{2}{*}{ Age } & \multicolumn{2}{|c|}{ Pneumonia $(-)(n=270)$} & \multicolumn{2}{|c|}{ Pneumonia $(+)(n=24)$} & \multirow{2}{*}{$\frac{p \text { value }}{0.29}$} \\
\hline & 69 & $69(32-85)$ & 71 & $(57-80)$ & \\
\hline \multicolumn{6}{|l|}{ Gender } \\
\hline Male & 211 & $(91.7 \%)$ & 19 & $(8.3 \%)$ & \multirow[t]{2}{*}{0.91} \\
\hline Female & 59 & $(92.2 \%)$ & 5 & $(7.8 \%)$ & \\
\hline \multicolumn{6}{|l|}{ Fibrosis staging } \\
\hline F $0-1$ & 98 & $(94.2 \%)$ & 6 & $(5.8 \%)$ & \multirow[t]{2}{*}{0.27} \\
\hline F 2-4 & 172 & $(90.5 \%)$ & 18 & $(9.5 \%)$ & \\
\hline \multicolumn{6}{|l|}{ Glucose intolerance } \\
\hline No & 179 & $(92.7 \%)$ & 14 & $(7.3 \%)$ & \multirow[t]{2}{*}{0.43} \\
\hline Yes & 91 & $(90.1 \%)$ & 10 & $(9.9 \%)$ & \\
\hline ICG-R15 (\%) & 13.9 & $(2.0-52.6)$ & 14.7 & $(3.6-48.1)$ & 0.27 \\
\hline Serum albumin (g/dl) & 3.9 & $(2.1-5.1)$ & 3.7 & $(2.8-4.6)$ & 0.02 \\
\hline Total bilirubin level (mg/dl) & 0.8 & $(0.1-2.2)$ & 0.7 & $(0.3-1.7)$ & 0.16 \\
\hline Prothrombin activity $(\%)$ & 88.2 & $(61.3-150)$ & 84.7 & $(53.8-121.5)$ & 0.27 \\
\hline Brinkman index & 400 & $(0-2200)$ & 800 & $(0-1600)$ & 0.02 \\
\hline $\mathrm{VC}(\mathrm{L})$ & 3.34 & $(1.28-6.12)$ & 2.97 & $(2.12-4.34)$ & 0.042 \\
\hline FEV1.0 (L) & 2.51 & $(0.99-4.82)$ & 2.22 & $(0.72-2.93)$ & $<0.01$ \\
\hline \multicolumn{6}{|l|}{ Sputum on POD1 } \\
\hline Without bacteria & 134 & $(98.5 \%)$ & 2 & $(1.5 \%)$ & \multirow[t]{2}{*}{$<0.01$} \\
\hline With bacteria & 136 & $(86.1 \%)$ & 22 & $(13.9 \%)$ & \\
\hline \multicolumn{6}{|l|}{ Type of hepatectomy } \\
\hline (Extended) hemihepatectomy or sectionectomy & 133 & $(92.4 \%)$ & 11 & $(7.6 \%)$ & \multirow[t]{2}{*}{0.75} \\
\hline Segmentectomy or partial hepatectomy & 137 & $(91.3 \%)$ & 13 & $(8.7 \%)$ & \\
\hline Operation time (min) & 358 & $(76-1028)$ & 328 & $(155-560)$ & 0.7 \\
\hline Blood loss (g) & 470 & $(13-9425)$ & 735 & $(35-2050)$ & 0.12 \\
\hline \multicolumn{6}{|l|}{ Mortality } \\
\hline No & 268 & $(92.7 \%)$ & 21 & $(7.3 \%)$ & \multirow[t]{2}{*}{$<0.01$} \\
\hline Yes & 2 & $(40.0 \%)$ & 3 & $(60.0 \%)$ & \\
\hline
\end{tabular}

Data are presented as absolute numbers (percentage) or median (range, minimum-maximum)

$H C C$ hepatocellular carcinoma, ICG-R15 indocyanine green retention rate at $15 \mathrm{~min}, V C$ vital capacity, FEV1.0 forced expiratory volume in $1.0 \mathrm{~s}$

patients) among Gram-negative bacteria and MRSA (8 patients) among Gram-positive bacteria, respectively. Bacterial homology in sputum obtained on POD1, and the day of onset of POP was noted in 13 (54.2\%) of the 24 patients with POP. In particular, there were 13 patients with POP caused by P. aeruginosa or MRSA, of whom homology was confirmed in 9 of those patients $(69.2 \%)$. The presence of bacteria in sputum on POD1 showed $91.7 \%$ sensitivity, $49.6 \%$ specificity, $13.9 \%$ positive predictive value, and $98.5 \%$ negative predictive value for prediction of POP. POP developed in only $2(1.5 \%)$ of 136 patients who expectorated sputum without bacteria, and in only $1(1.4 \%)$ patient among 69 patients who did not expectorate sputum on POD1.

Comparison of perioperative factors between groups with and without POP revealed a significantly increased risk of POP in patients in association with serum albumin $(p=0.02)$, Brinkman index $(p=0.02), \mathrm{VC}(p=0.042), \mathrm{FEV} 1.0(p<0.01)$, and sputum with bacteria on POD1 $(p<0.01)$, respectively. POP significantly increased mortality ( 0.74 vs $12.5 \%$, $p<0.01$ ) (Table 3). ROC curve analysis indicated that the optimal cut-offs for serum albumin, the Brinkman index, VC,

Table 4 Multivariate logistic regression predicting development of postoperative pneumonia

\begin{tabular}{lrrr}
\hline Factor & OR & $95 \%$ CI & $p$ value \\
\hline Serum albumin $(\mathrm{g} / \mathrm{dl})(<3.9$ vs. $\geq 4.0)$ & 2.88 & $0.98-8.52$ & 0.06 \\
Brinkman index $(\geq 400$ vs. $<400)$ & 4.29 & $1.44-12.8$ & $<0.01$ \\
VC $(<3.18$ vs. $\geq 3.18)$ & 1.88 & $0.55-6.36$ & 0.31 \\
FEV1.0 $(<2.44$ vs. $\geq 2.44)$ & 2.87 & $0.79-10.4$ & 0.11 \\
Bacteria in sputum on POD1 (yes vs. no) & 9.43 & $2.11-42.0$ & $<0.01$
\end{tabular}

$O R$ odds ratios, $C I$ confidence interval, $V C$ vital capacity, FEV1.0 forced expiratory volume in $1.0 \mathrm{~s}, P O D$ postoperative day 
and FEV1.0 were $4.0 \mathrm{~g} / \mathrm{dL}(79.2 \%$ sensitivity and $47.8 \%$ specificity), 400 (79.2\% sensitivity and $48.9 \%$ specificity), $3.18 \mathrm{~L}$ ( $66.7 \%$ sensitivity and $60.4 \%$ specificity), and $2.44 \mathrm{~L}$ ( $75.0 \%$ sensitivity and $54.1 \%$ specificity), respectively, for the occurrence of POP. Multivariate analysis demonstrated that Brinkman index $\geq 400$ and bacteria in sputum on POD1 were independent predictors of POP (Table 4).

\section{Discussion}

In the current study, the incidence of POP after hepatectomy was $8.2 \%$. In addition, patients with POP had significantly increased mortality. Although the homology between species bacteria in sputum on POD1 and caused pneumonia was not high (54\%), sensitivity (91.7\%) and negative predictive value (98.5\%) of the presence of bacteria in sputum on POD1 to predict POP were high. According to multivariate analysis, two independent risk factors for POP after hepatectomy were statistically significant: Brinkman index $\geq 400$ and bacteria in sputum on POD1. Although several risk factors have already been reported for the occurrence of pulmonary complications, ${ }^{4,6,16,17}$ the risk factors have varied between studies due to differences in the study objectives, the definition used for pulmonary complications, and the type of surgery.

Previous studies have shown that the risk of postoperative pulmonary complications is mildly elevated among smokers. ${ }^{18,19}$ In the current study, $67.3 \%$ of the patients had a history of smoking, and about half of them had a Brinkman index $\geq 400$. Kojima et al. reported that a Brinkman index $\geq 400$ relates to a risk for decreased respiratory function. ${ }^{20} \mathrm{In}$ the field of esophageal surgery, smoking has been reported to be a risk factor for pulmonary complications, and it has also been reported that tobacco cessation and preoperative respiratory rehabilitation are expected to reduce the occurrence of complications. Two randomized trials have studied the impact of perioperative smoking intervention programs. ${ }^{21,22}$ Because both trials studied patients undergoing low-risk procedures, the trials were insufficiently powered to show a difference in pulmonary complication rates. A previous cohort study showed paradoxically higher postoperative pulmonary complication rates for smokers who stopped or reduced smoking within 2 months before noncardiothoracic surgery. ${ }^{23}$ Preoperative cigarette cessation to reduce the occurrence of pulmonary complications has been controversial.

Carrel et al. reported the relationship between preoperative tracheal aspirates of patients undergoing CABG and POP. ${ }^{10}$ Of 500 patients, $91(18.2 \%)$ had a positive Gram stain, and the incidence of POP was significantly higher in patients with preoperative positive tracheal aspirates $(15.3 \%)$ than in patients with negative tracheal aspirates $(3.6 \% ; p<0.01)$. In patients experiencing POP, there was a high correlation between the bacteria in preoperative aspirates and the bacteria observed when POP developed. A positive tracheal aspirate was one of the significant risk factors for developing postoperative pneumonia. In comparing the present study and the report of Carrel et al., although there is no difference in the incidence of POP in the patients who had bacteria in their sputum on POD1 (22/158: $13.9 \%$ ), the incidence of bacteria-positive sputum on POD1 (158/294: $53.7 \%$ ) was higher in the present study than in the Carrel report. The sputum specimens on POD1 may have been affected by surgical stress or intraoperative antibiotic prophylaxis. Conversely, we believe there is significance to the sample collection after the bacterial flora has been changed by these modifications. When the presence of bacteria in sputum on POD1 was confirmed and POP subsequently developed, this information allowed for the selection of an appropriate therapeutic antimicrobial drug against $P O P$.

In conclusion, the current study examined the frequency of POP after hepatectomy and investigated the risk factors for POP. The patients with a Brinkman index of $\geq 400$ and bacteria in sputum on POD1 require careful pulmonary care and should be closely monitored during the perioperative period. The information obtained from the patient's sputum on POD1 may be useful in the treatment of POP. We did not have any protocol to prevent POP against carrier having bacteria in sputum on POD1 in this study. The protocol that prophylactic antibiotics for POP is administered using the information of bacteria in sputum on POD1 may be considered prospectively.

Conflict of Interest The authors declare that they have no competing interests.

Open Access This article is distributed under the terms of the Creative Commons Attribution 4.0 International License (http:// creativecommons.org/licenses/by/4.0/), which permits unrestricted use, distribution, and reproduction in any medium, provided you give appropriate credit to the original author(s) and the source, provide a link to the Creative Commons license, and indicate if changes were made.

\section{References}

1. Lawrence VA, Hilsenbeck SG, Mulrow CD, et al. Incidence and hospital stay for cardiac and pulmonary complications after abdominal surgery. J Gen Intern Med. 1995; 10(12): 671-8.

2. Smetana GW. Preoperative pulmonary evaluation. N Engl J Med. 1999; 340(12): 937-44.

3. Barisione G, Rovida S, Gazzaniga GM, Fontana L. Upper abdominal surgery: does a lung function test exist to predict early severe postoperative respiratory complications? Eur Respir J. 1997; 10(6): 1301-8.

4. Schroeder RA, Marroquin CE, Bute BP, et al. Predictive indices of morbidity and mortality after liver resection. Ann Surg. 2006; 243(3): 373-9.

5. Mathur AK, Ghaferi AA, Sell K, et al. Influence of body mass index on complications and oncologic outcomes following hepatectomy for malignancy. J Gastrointest Surg. 2010; 14(5): 849-57. 
6. Nobili C, Marzano E, Oussoultzoglou E, et al. Multivariate analysis of risk factors for pulmonary complications after hepatic resection. Ann Surg. 2012; 255(3): 540-50.

7. Pessaux P, van den Broek MA, Wu T, et al. Identification and validation of risk factors for postoperative infectious complications following hepatectomy. J Gastrointest Surg. 2013; 17(11): 1907-16.

8. Smetana GW. Preoperative pulmonary evaluation: identifying and reducing risks for pulmonary complications. Cleve Clin J Med. 2006; 73 Suppl 1: S36-41.

9. Lawrence VA, Cornell JE, Smetana GW. Strategies to reduce postoperative pulmonary complications after noncardiothoracic surgery: systematic review for the American College of Physicians. Ann Intern Med. 2006; 144(8): 596-608.

10. Carrel TP, Eisinger E, Vogt M, Turina MI. Pneumonia after cardiac surgery is predictable by tracheal aspirates but cannot be prevented by prolonged antibiotic prophylaxis. Ann Thorac Surg. 2001; 72(1): 143-8.

11. Brinkman GL, Coates EO, Jr. The effect of bronchitis, smoking, and occupation on ventilation. The American review of respiratory disease. 1963; 87: 684-93.

12. Takao T, Nishida M, Maeda Y, et al. Effect of cautery with irrigation forceps on the remnant liver after hepatectomy in rats. Eur Surg Res. 1999; 31(2): 173-9.

13. Hirokawa F, Hayashi M, Miyamoto Y, et al. A novel method using the VIO soft-coagulation system for liver resection. Surgery. 2011; 149(3): 438-44.

14. Itoh S, Fukuzawa K, Shitomi Y, et al. Impact of the VIO system in hepatic resection for patients with hepatocellular carcinoma. Surg Today. 2012; 42(12): 1176-82.
15. Bedossa P, Poynard T. An algorithm for the grading of activity in chronic hepatitis C. The METAVIR Cooperative Study Group. Hepatology. 1996; 24(2): 289-93.

16. McAlister FA, Bertsch K, Man J, Bradley J, Jacka M. Incidence of and risk factors for pulmonary complications after nonthoracic surgery. Am J Respir Crit Care Med. 2005; 171(5): 514-7.

17. Kanat F, Golcuk A, Teke T, Golcuk M. Risk factors for postoperative pulmonary complications in upper abdominal surgery. ANZ J Surg. 2007; 77(3): 135-41.

18. Qaseem A, Snow V, Fitterman N, et al. Risk assessment for and strategies to reduce perioperative pulmonary complications for patients undergoing noncardiothoracic surgery: a guideline from the American College of Physicians. Ann Intern Med. 2006; 144(8): 575-80.

19. Smetana GW. Postoperative pulmonary complications: an update on risk assessment and reduction. Cleve Clin J Med. 2009; 76(4): 60-5.

20. Kojima S, Sakakibara H, Motani S, et al. Incidence of chronic obstructive pulmonary disease, and the relationship between age and smoking in a Japanese population. J Epidemiol. 2007; 17(2): 54-60.

21. Moller AM, Villebro N, Pedersen T, Tonnesen H. Effect of preoperative smoking intervention on postoperative complications: a randomised clinical trial. Lancet. 2002; 359(9301): 114-7.

22. Lindstrom D, Sadr Azodi O, Wladis A, et al. Effects of a perioperative smoking cessation intervention on postoperative complications: a randomized trial. Ann Surg. 2008; 248(5): 739-45.

23. Bluman LG, Mosca L, Newman N, Simon DG. Preoperative smoking habits and postoperative pulmonary complications. Chest. 1998; 113(4): 883-9. 